\title{
On the stable set of an analytic gradient flow
}

\author{
by Zbigniew Szafraniec
}

\begin{abstract}
Let $f: \mathbb{R}^{n} \rightarrow \mathbb{R}, n \geq 2$, be a real analytic function. In this paper we study the stable set of the gradient flow $\dot{x}=\nabla f(x)$ associated with a critical point of $f$. In particular we present simple topological conditions which imply that this set contains an infinite family of trajectories, or has a non-empty interior.
\end{abstract}

\section{Introduction.}

Let $f: \mathbb{R}^{n} \rightarrow \mathbb{R}, n \geq 2$, be an analytic function. According to Łojasiewicz [16], the limit set of a trajectory of the dynamical system $\dot{x}=\nabla f(x)$ is either empty or contains a single critical point of $f$. So the family of integral curves which converge to a critical point is a natural object of study in the theory of gradient dynamical systems.

Let $f: \mathbb{R}^{n}, 0 \rightarrow \mathbb{R}, 0$ be an analytic function defined in a neighbourhood of the origin, having a critical point at 0 . We shall write $T(f)$ for the set of integral curves which converge to the origin, and $S(f)$ for the stable set of the origin, which is the union of all orbits of the solutions that converge to the origin. By [16], the stable set in closed near the origin.

In this paper we study the naturally occurring question whether the set $T(f)$ is infinite or whether the interior of $S(f)$ is non-empty? (In the planar case these problems are equivalent.) By Remark 5.1, if $T(f)$ is infinite then it has the cardinality of the continuum.

In some cases the answer is rather obvious. If the hessian matrix of $f$ at the origin has at least two negative eigenvalues then the dimension of the stable manifold at the origin is $\geq 2$, and then $T(f)$ is infinite. If the origin is a local strict maximum then int $S(f) \neq \emptyset$. It is worth pointing out that according to Moussu [18, Theorem 3] the family $T(f)$ always contain trajectories which are represented by real analytic curves converging to the origin. In some cases the family of those analytic curves can be infinite.

Let $\omega: \mathbb{R}^{n}, 0 \rightarrow \mathbb{R}, 0$ be the homogeneous initial form associated with $f$. Put $\Omega=S^{n-1} \cap\{\omega<0\}$. Applying the Moussu results [18] one may show that $\operatorname{dim} S(f) \geq 2$ if there exists at least one non-degenerate critical point of $\omega \mid \Omega$ which is not a local minimum. In that case the set $T(f)$ is infinite. 
Moreover, if there exists at least one non-degenerate critical point of $\omega \mid \Omega$ which is a local maximum then int $S(f) \neq \emptyset$.

Let $S_{r}=S_{r}^{n-1} \cap\{f<0\}$, where $S_{r}^{n-1}=\left\{x \in \mathbb{R}^{n}|| x \mid=r\right\}, 0<r \ll 1$. By [19], [20], if $T(f)$ is finite then each cohomology group $H^{i}\left(S_{r}\right)$ is trivial for $i \geq 1$. Hence, if there exists $i \geq 1$ with $H^{i}\left(S_{r}\right) \neq 0$, then $T(f)$ is infinite.

However, there are examples where $n=2$ and none of the above assumptions holds but $T(f)$ is infinite (see Example 5.12 ).

In the course of proving Thom's gradient conjecture Kurdyka et al. 12 . have applied advanced techniques of analytic geometry so as to investigate geometric properties of trajectories converging to a the origin. In particular they have proved that with each such a trajectory one can associate a pair $(\ell, a) \in L^{\prime}(f)$, where $\ell$ is a characteristic exponent of $f$, the number $a$ is an asymptotic critical value of $f /|x|^{\ell}$, and $L^{\prime}(f)$ is a finite subset of $\mathbb{Q}^{+} \times \mathbb{R}_{-}$, where $\mathbb{Q}^{+}$is the set of positive rationals and $\mathbb{R}_{-}$is the set of negative real numbers.

In [4] (see also Section 4) there is presented an intrinsic filtration of $T(f)$ given in terms of characteristic exponents and asymptotic critical values of $f$. Unfortunately, these numbers are difficult to compute. This is why in this paper we present methods which are more easy to apply.

The first main result of this paper shows that $T(f)$ is infinite if rank $H^{0}\left(S_{r}\right)<$ rank $H^{0}(\Omega)$ (see Theorem 5.10), i.e. if $S_{r}$ has less connected components than $\Omega$. As a corollary we shall show that the inequality $\chi\left(S_{r}\right)<\chi(\Omega)$ implies that $T(f)$ is infinite. It is proper to add that there exist efficient methods of computing those Euler-Poincaré characteristics (see [15], 22]). (These results have been earlier presented in [23].)

The second main result of this paper shows that int $S(f) \neq \emptyset$ if rank $H^{n-2}\left(S_{r}\right)<$ rank $H^{n-2}(\Omega)$, where $H^{n-2}(\cdot)$ is the $(n-2)$-th cohomology group with rational coefficients (see Theorem 6.2).

Let $\Omega^{\prime}=S^{n-1} \cap\{\omega \geq 0\}=S^{n-1} \backslash \Omega$, and $S_{r}^{\prime}=S_{r}^{n-1} \cap\{f \geq 0\}=S_{r}^{n-1} \backslash S_{r}$, $0<r \ll 1$. Sets $\Omega^{\prime}, S_{r}^{\prime}$ are compact and semianalytic, hence they are triagulable. By the Alexander duality theorem, if $S_{r}^{\prime}$ and $\Omega^{\prime}$ are non-empty then $\operatorname{rank} H_{0}\left(S_{r}^{\prime}\right)=1+\operatorname{rank} H^{n-2}\left(S_{r}\right)$ and $\operatorname{rank} H_{0}\left(\Omega^{\prime}\right)=1+\operatorname{rank} H^{n-2}(\Omega)$. Thus, if $S_{r}^{\prime}$ has less connected components than $\Omega^{\prime}$ then the interior of $S(f)$ is non-empty (see Theorem 6.3).

Let $f$ be as above. Assume that $g: \mathbb{R}^{n}, 0 \rightarrow \mathbb{R}, 0$ an analytic function which is right-equivalent to $f$. We shall prove that $T(g)$ is infinite (resp. int $S(g) \neq \emptyset$ ) if $S_{r}$ has less connected components than $\Omega$ (resp. if $S_{r}^{\prime}$ has less connected components than $\Omega^{\prime}$ ) (see Theorem 7.1 ). 
The paper is organized as follows. In Section 2 we prove sufficient conditions which imply that a compact subset of the sphere has a non-empty interior. In Section 3 we study the homotopy type of some semi-analytic sets. In Section 4 we present properties of important geometric invariants associated with trajectories of the gradient flow. In Sections 5, 6, we prove the main results (Theorems 5.10, 6.2, 6.3). Section 7 is devoted to functions right-equivalent to the ones that satisfy assumptions of those theorems. References [1, 2, 6, 12, 13, 14, 20] present significant related results and applications.

\section{Sets with non-empty interior.}

In this section we present some consequences of the Alexander duality theorem. The best reference here is [21].

Lemma 2.1. Suppose that $L \subset K$ are closed subsets of $S^{n-1}, n \geq 2$, and $\operatorname{rank} \bar{H}^{n-2}(K)<\operatorname{rank} \bar{H}^{n-2}(L)<\infty$, where $\bar{H}^{n-2}(\cdot)$ is the $(n-2)$-th $\check{C}$ echAlexander-Spanier cohomology group with rational coefficients. Then the interior of $K$ is non-empty.

Proof. As $\bar{H}^{n-2}(L) \neq 0$ then sets $L, K, S^{n-1} \backslash L$ are not void. If $K=S^{n-1}$ then the assertion holds. From now on we assume that $S^{n-1} \backslash K \neq \emptyset$ and $n \geq 3$.

By the Alexander duality theorem there are isomorphisms

$$
\bar{H}^{n-2}(L) \simeq \tilde{H}_{0}\left(S^{n-1} \backslash L\right), \bar{H}^{n-2}(K) \simeq \tilde{H}_{0}\left(S^{n-1} \backslash K\right),
$$

where $\tilde{H}_{0}(\cdot)$ is the 0 -th reduced singular homology group with rational coefficients.

Then $S^{n-1} \backslash L$ is a disjoint union of open connected components $U_{1}, \ldots, U_{\ell}$, where $\ell=1+\operatorname{rank} \tilde{H}_{0}\left(S^{n-1} \backslash L\right)=1+\operatorname{rank} \bar{H}^{n-2}(L)$, and $S^{n-1} \backslash K$ is a disjoint union of open connected components $V_{1}, \cdots, V_{k}$, where $k=1+$ $\operatorname{rank} \tilde{H}_{0}\left(S^{n-1} \backslash K\right)=1+\operatorname{rank} \bar{H}^{n-2}(K)$.

Suppose that $U_{i} \backslash K \neq \emptyset$ for each $1 \leq i \leq \ell$, so that there are points $p_{i} \in U_{i} \backslash K$ and then $p_{i} \in V_{j(i)}$ for some $1 \leq j(i) \leq k$. As $V_{j(i)}$ is a connected subset of $U_{1} \cup \ldots \cup U_{\ell}$, then $V_{j(i)} \subset U_{i}$.

Because $U_{i}$ are pairwise disjoint, then $V_{j(i)}$ are pairwise disjoint too. Hence $k \geq \ell$, contrary to our claim. Then at least one open connected component $U_{i}$ is a subset of $K$. 
Similar arguments apply to the case where $n=2$.

Corollary 2.2. Suppose that $L \subset K \subset F$, where $L, K$ are compact, $n \geq$ 2 , $\operatorname{rank} \bar{H}^{n-2}(K)<\operatorname{rank} \bar{H}^{n-2}(L)<\infty$, and $F$ is an $(n-1)$-dimensional manifold homeomorphic to a subset of $S^{n-1}$. Then the interior of $K$ is nonempty.

\section{Homotopy type of semi-analytic sets.}

Let $f: \mathbb{R}^{n}, 0 \rightarrow \mathbb{R}, 0$ be an analytic function defined in an open neighbourhood of the origin. Let $\mathbb{Q}^{+}$denote the set of positive rationals. For $\ell \in \mathbb{Q}^{+}$, $a<0, y<0$ and $r>0$ we shall write

$$
\begin{gathered}
B_{r}^{n}=\left\{x \in \mathbb{R}^{n}|| x \mid \leq r\right\}, \quad S_{r}^{n-1}=\left\{x \in \mathbb{R}^{n}|| x \mid=r\right\}, \\
V^{\ell, a}=\left\{\left.x \in \mathbb{R}^{n} \backslash\{0\}|f(x) \leq a| x\right|^{\ell}\right\}, \\
S_{r}^{\ell, a}=S_{r}^{n-1} \cap V^{\ell, a}, \quad B_{r}^{\ell, a}=B_{r}^{n} \cap V^{\ell, a}, \quad F^{\ell, a}(y)=f^{-1}(y) \cap V^{\ell, a}, \\
D^{\ell, a}(y)=f^{-1}([y, 0)) \cap V^{\ell, a}=\left\{x \in V^{\ell, a} \mid y \leq f(x)<0\right\} .
\end{gathered}
$$

Lemma 3.1. Assume that $\ell \in \mathbb{Q}^{+}$and $a<0$. If $0<-y \ll r \ll 1$ then the sets $S_{r}^{\ell, a}$ and $F^{\ell, a}(y)$ are homotopy equivalent. In particular, the singular cohomology groups $H^{*}\left(S_{r}^{\ell, a}\right)$ and $H^{*}\left(F^{\ell, a}(y)\right)$ are isomorphic.

Proof. For $x \in V^{\ell, a} \cup\{0\}$ lying sufficiently close to the origin we have $|x|^{1 / 2} \geq$ $|f(x)| \geq|a| \cdot|x|^{\ell}$, so that in particular functions $f(x),|x|^{2}$ restricted to this set are proper. According to the local triviality of proper analytic mappings between semi-analytic sets (see [7, 8, 9]), they are locally trivial. So there is $r_{0}>0$ such that $|x|: B_{r_{0}}^{\ell, a} \rightarrow\left(0, r_{0}\right]$ is a trivial fibration. Hence the inclusion $S_{r}^{\ell, a} \subset B_{r}^{\ell, a}$ is a homotopy equivalence for each $0<r \leq r_{0}$.

By similar arguments, there is $y_{0}<0$ such that $D^{\ell, a}\left(y_{0}\right) \subset B_{r_{0}}^{\ell, a}$ and $f$ : $D^{\ell, a}\left(y_{0}\right) \rightarrow\left[y_{0}, 0\right)$ is a trivial fibration, so that the inclusion $F^{\ell, a}(y) \subset D^{\ell, a}(y)$ is a homotopy equivalence for each $y_{0} \leq y<0$.

So, if $0<-y \ll r \ll 1$ then we may assume that $r \leq r_{0}, y_{0} \leq y$, and

$$
D^{\ell, a}(y) \subset B_{r}^{\ell, a} \subset D^{\ell, a}\left(y_{0}\right) \subset B_{r_{0}}^{\ell, a} .
$$

As inclusions $D^{\ell, a}(y) \subset D^{\ell, a}\left(y_{0}\right)$ and $B_{r}^{\ell, a} \subset B_{r_{0}}^{\ell, a}$ are homotopy equivalencies, then $D^{\ell, a}(y) \subset B_{r}^{\ell, a}$ is a homotopy equivalence too. Then $F^{\ell, a}(y)$ is homotopy equivalent to $S_{r}^{\ell, a}$. 
For $0<-y \ll r \ll 1$ we shall write

$$
F_{r}(y)=B_{r}^{n} \cap f^{-1}(y), \quad S_{r}=\left\{x \in S_{r}^{n-1} \mid f(x)<0\right\} .
$$

We call the set $F_{r}(y)$ the real Milnor fibre. According to [17], it is either an $(n-1)$-dimensional compact manifold with boundary or an empty set. Moreover, the sets $F_{r}(y)$ and $S_{r}$ are homotopy equivalent.

Corollary 3.2. If $0<-y \ll r \ll 1$ then the cohomology groups $H^{*}\left(S_{r}\right)$ and $H^{*}\left(F_{r}(y)\right)$ are isomorphic.

Lemma 3.3. If $\ell \in \mathbb{Q}^{+}, a<0$ and $0<-y \ll r \ll 1$ then $F^{\ell, a}(y)=\{x \in$ $\left.\left.F_{r}(y)|y \leq a| x\right|^{\ell}\right\}$. In particular, $F^{\ell, a}(y) \subset F_{r}(y)$.

Proof. If $x \in F^{\ell, a}(y)$ then $y=f(x) \leq a|x|^{\ell}$, and then $|x|^{\ell} \leq y / a$. If $0<$ $-y \ll r \ll 1$ then $|x| \leq r$, and then $x \in B_{r}^{n} \cap f^{-1}(y)=F_{r}(y)$.

If $x \in B_{r}^{n} \cap f^{-1}(y)$ and $y \leq a|x|^{\ell}$, then $x \in f^{-1}(y) \cap V^{\ell, a}=F^{\ell, a}(y)$. Hence, if $0<-y \ll r \ll 1$ then $\left\{\left.x \in F_{r}(y)|y \leq a| x\right|^{\ell}\right\} \subset F^{\ell, a}(y)$.

Let $\omega$ be the initial form associated with $f$ and let $g=f-\omega$, so that $f=\omega+g$. Denote by $d$ the degree of $\omega$. Hence $g=O\left(|x|^{d+1}\right)$.

Lemma 3.4. If $0<r \ll-a \ll 1$ then sets $S_{r}^{d, a}=S_{r}^{n-1} \cap\left\{f \leq a r^{d}\right\}$, $S_{r}^{n-1} \cap\left\{\omega \leq a r^{d}\right\}$ and $\Omega=S^{n-1} \cap\{\omega<0\}$ have the same homotopy type.

Proof. For $r \in \mathbb{R}$ sufficiently close to zero and $x \in S^{n-1}$ we have

$$
f(r x)=\omega(r x)+g(r x)=r^{d} \omega(x)+r^{d+1} G(x, r),
$$

where $G(x, r)$ is an analytic function defined in an open neighbourhood of $S^{n-1} \times\{0\}$. Put $H(x, r)=\omega(x)+r G(x, r)$, and $H_{r}=H(\cdot, r): S^{n-1} \rightarrow \mathbb{R}$.

By [17, Corollary 2.8], there exists $a_{0}<0$ such that any $a_{0}<a<0$ is a regular value of $\omega \mid S^{n-1}$. Hence there exists $r_{0}>0$ such that $a$ is a regular value of every $H_{r}$, where $-r_{0}<r<r_{0}$. Then

$$
\left\{(x, r) \in S^{n-1} \times\left(-r_{0}, r_{0}\right) \mid H(x, r) \leq a\right\}
$$

is an $n$-dimensional manifold with boundary $S^{n-1} \times\left(-r_{0}, r_{0}\right) \cap H^{-1}(a)$. By the implicit function theorem, the mapping $(x, r) \mapsto r$ restricted to both above manifolds is a proper submersion. By Ehresmann's theorem, it is a locally trivial fibration. Hence if $r$ is sufficiently close to zero then the manifolds 
$S^{n-1} \cap\{\omega \leq a\}=\left\{x \in S^{n-1} \mid H(x, 0) \leq a\right\}$ and $S^{n-1} \cap\left\{H_{r} \leq a\right\}=\{x \in$ $\left.S^{n-1} \mid H(x, r) \leq a\right\}$ are homeomorphic.

The set $S^{n-1} \cap\{\omega \leq a\}$ is a deformation retract of $\Omega=S^{n-1} \cap\{\omega<0\}$, so that these sets have the same homotopy type.

We have $f(r x)=r^{d} H_{r}(x)$. Hence $x \in S^{n-1} \cap\left\{H_{r} \leq a\right\}$ if and only if $r x \in S_{r}^{n-1} \cap\left\{f \leq a r^{d}\right\}$, and the proof is complete.

\section{Geometric properties of trajectories.}

In the beginning of this section we present some results obtained by Kurdyka et al. [12], [13] in the course of proving Thom's gradient conjecture. In exposition and notation we follow closely these papers.

Let $f: \mathbb{R}^{n}, 0 \rightarrow \mathbb{R}, 0$ be an analytic function defined in a neighbourhood of the origin, having a critical point at 0 . The gradient $\nabla f(x)$ splits into its radial component $\frac{\partial f}{\partial r}(x) \frac{x}{|x|}$ and the spherical one $\nabla^{\prime} f(x)=\nabla f(x)-\frac{\partial f}{\partial r}(x) \frac{x}{|x|}$. We shall denote $\frac{\partial f}{\partial r}$ by $\partial_{r} f$.

For $\epsilon>0$ define $W^{\epsilon}=\left\{x|f(x) \neq 0, \epsilon| \nabla^{\prime} f|\leq| \partial_{r} f \mid\right\}$. There exists a finite subset of positive rationals $L(f) \subset \mathbb{Q}^{+}$such that for any $\epsilon>0$ and any sequence $W^{\epsilon} \ni x \rightarrow 0$ there is a subsequence $W^{\epsilon} \ni x^{\prime} \rightarrow 0$ and $\ell \in L(f)$ such that

$$
\frac{\left|x^{\prime}\right| \partial_{r} f\left(x^{\prime}\right)}{f\left(x^{\prime}\right)} \rightarrow \ell
$$

Elements of $L(f)$ are called characteristic exponents.

Fix $\ell>0$, not necessarily in $L(f)$, and consider $F=f /|x|^{\ell}$ defined in the complement of the origin. We say that $a \in \mathbb{R}$ is an asymptotic critical value of $F$ at the origin if there exists a sequence $x \rightarrow 0, x \neq 0$, such that

$$
|x| \cdot|\nabla F(x)| \rightarrow 0 \quad, \quad F(x)=\frac{f(x)}{|x|^{\ell}} \rightarrow a .
$$

The set of asymptotic critical values of $F$ is finite.

The real number $a \neq 0$ is an asymptotic critical value if and only if there exists a sequence $x \rightarrow 0, x \neq 0$, such that

$$
\frac{\left|\nabla^{\prime} f(x)\right|}{\left|\partial_{r} f(x)\right|} \rightarrow 0 \quad, \quad \frac{f(x)}{|x|^{\ell}} \rightarrow a \text {. }
$$

Hence the set

$L^{\prime}(f)=\left\{(\ell, a) \mid \ell \in L(f), a<0\right.$ is an asymptotic critical value of $\left.f /|x|^{\ell}\right\}$ 
is a finite subset of $\mathbb{Q}^{+} \times \mathbb{R}_{-}$, where $\mathbb{R}_{-}$is the set of negative real numbers.

We shall write $T(f)$ for the set of non-trivial trajectories of the gradient flow $\dot{x}=\nabla f(x)$ converging to the origin. By Section 6 of [12], for every such a trajectory $x(t)$, with $x(t) \rightarrow 0$, there exists a unique pair $\left(\ell^{\prime}, a^{\prime}\right) \in L^{\prime}(f)$ such that $f(x(t)) /|x(t)|^{\ell^{\prime}} \rightarrow a^{\prime}$. There is a natural partition of $T(f)$ associated with $L^{\prime}(f)$. Namely for $\left(\ell^{\prime}, a^{\prime}\right) \in L^{\prime}(f)$,

$$
T^{\ell^{\prime}, a^{\prime}}(f)=\left\{\left.x(t) \in T(f)|f(x(t)) /| x(t)\right|^{\ell^{\prime}} \rightarrow a^{\prime} \text { as } x(t) \rightarrow 0\right\} .
$$

In the set $\mathbb{Q}^{+} \times \mathbb{R}_{-}$we can introduce the lexicographic order

$$
\left(\ell^{\prime}, a^{\prime}\right) \leq(\ell, a) \text { if } \ell^{\prime}<\ell \text {, or } \ell^{\prime}=\ell \text { and } a^{\prime} \leq a .
$$

Take $(\ell, a) \in \mathbb{Q}^{+} \times \mathbb{R}_{-} \backslash L^{\prime}(f)$. We shall write

$$
\tilde{T}^{\ell, a}(f)=\bigcup T^{\ell^{\prime}, a^{\prime}}(f) \text {, where }\left(\ell^{\prime}, a^{\prime}\right)<(\ell, a) \text { and }\left(\ell^{\prime}, a^{\prime}\right) \in L^{\prime}(f) .
$$

According to [19], there are $0<-y \ll r \ll 1$ such that each trajectory $x(t) \in T(f)$ intersects $F_{r}(y)$ transversally at exactly one point. Let $\Gamma(f) \subset$ $F_{r}(y)$ be the union of all those points. By [16] the set $\Gamma(f)$ is closed subset of $F_{r}(y)$, hence it is compact. So there is a natural one-to-one correspondence between trajectories in $T(f)$ and points in $\Gamma(f)$. The same way one can define the set $\Gamma^{\ell^{\prime}, a^{\prime}}(f) \subset F_{r}(y)$ (resp. $\left.\tilde{\Gamma}^{\ell, a}(f) \subset F_{r}(y)\right)$ whose points are in one-to-one correspondence with trajectories from $T^{\ell^{\prime}, a^{\prime}}(f)$ (resp. $\left.\tilde{T}^{\ell, a}(f)\right)$. In particular, for $(\ell, a) \in \mathbb{Q}^{+} \times \mathbb{R}_{-} \backslash L^{\prime}(f)$ the set

$$
\tilde{\Gamma}^{\ell, a}(f)=\bigcup \Gamma^{\ell^{\prime}, a^{\prime}}(f) \text {, where }\left(\ell^{\prime}, a^{\prime}\right)<(\ell, a) \text { and }\left(\ell^{\prime}, a^{\prime}\right) \in L^{\prime}(f),
$$

is a subset of $\Gamma(f)$.

By [19, Theorem 12], [4, Theorem 6] and Lemma 3.3 we have

Theorem 4.1. If $0<-y \ll r \ll 1$ then the inclusion $\Gamma(f) \subset F_{r}(y)$ induces an isomorphism

$$
\bar{H}^{*}(\Gamma(f)) \simeq H^{*}\left(F_{r}(y)\right),
$$

where $\bar{H}^{*}(\cdot)$ is the Čech-Alexander-Spanier cohomology group and $H^{*}(\cdot)$ is the singular cohomology group.. In particular $\Gamma(f)$ has the same (finite) number of connected components as $F_{r}(y)$.

Moreover, for every $(\ell, a) \in \mathbb{Q}^{+} \times \mathbb{R}_{-} \backslash L^{\prime}(f)$ the set $\tilde{\Gamma}^{\ell, a}(f)$ is a compact subset of $F^{\ell, a}(y)$. The inclusion induces an isomorphism

$$
\bar{H}^{*}\left(\tilde{\Gamma}^{\ell, a}(f)\right) \simeq H^{*}\left(F^{\ell, a}(y)\right) .
$$




\section{Cardinality of $T(f)$}

The cardinality of the set $T(f)$ is obviously the same as that of $\Gamma(f)$. In this section we shall present simple topological conditions which imply that $\Gamma(f)$ and $T(f)$ are infinite sets.

We shall write $S(f)$ for the stable set of the origin, which is the union of all orbits of the solutions that converge to the origin.

Remark 5.1. If $\Gamma(f)$ is infinite then it contains at least one compact and infinite connected component, which is obviously not a zero-dimensional space. If that is the case then the Menger-Urysohn dimension as well as the CechLebesgue covering dimension of this component is at least one (see [5]), sets $\Gamma(f)$ and $T(f)$ have the cardinality of the continuum, and the dimension of the stable set $S(f)$ is at least two.

By Lemma 3.1, Corollary 3.2 and Theorem 4.1 we get

Corollary 5.2. There is an isomorphism $\bar{H}^{*}(\Gamma(f)) \simeq H^{*}\left(S_{r}\right)$. In particular $\Gamma(f)$ has the same (finite) number of connected components as $S_{r}$. If there exists $i \geq 1$ such that $H^{i}\left(S_{r}\right) \neq 0$ then $T(f)$ is infinite. So, if $S_{r} \neq \emptyset$ and the Euler-Poincaré characteristic $\chi\left(S_{r}\right) \leq 0$, then $T(f)$ is infinite.

Moreover, for every $(\ell, a) \in \mathbb{Q}^{+} \times \mathbb{R}_{-} \backslash L^{\prime}(f)$, if $0<r \ll 1$ then

$$
\bar{H}^{*}\left(\tilde{\Gamma}^{\ell, a}(f)\right) \simeq H^{*}\left(S_{r}^{\ell, a}\right)
$$

Example 5.3. The polynomial $f(x, y, z)=x^{3}+x^{2} z-y^{2}$ is weighted homogeneous. Of course $S_{r} \neq \emptyset$. By [22, p.245], the Euler-Poincaré characteristic $\chi\left(S_{r}^{2} \cap\{f \geq 0\}\right)=2$. By the Alexander duality theorem we have $\chi\left(S_{r}\right)=0$. Hence the set $T(f)$ is infinite.

Proposition 5.4. If $0<-a \ll 1$ then $\bar{H}^{*}\left(\tilde{\Gamma}^{d, a}(f)\right) \simeq H^{*}(\Omega)$. If $H^{i}(\Omega) \neq 0$ for some $i \geq 1$ then $T(f)$ is infinite.

Proof. As $L^{\prime}(f)$ is finite, if $0<-a \ll 1$ then $(d, a) \notin L^{\prime}(f)$. By Corollary 5.2 and Lemma [3.4, if $0<r \ll-a$ then we have

$$
\bar{H}^{*}\left(\tilde{\Gamma}^{d, a}(f)\right) \simeq H^{*}\left(S_{r}^{d, a}\right) \simeq H^{*}(\Omega) .
$$

In particular, if $H^{i}(\Omega) \neq 0$ for some $i \geq 1$ then $\tilde{\Gamma}^{d, a}(f)$ is infinite. Hence $\tilde{T}^{d, a}(f)$, as well as $T(f)$, is infinite. 
Example 5.5. Let $f(x, y, z)=z\left(x^{2}+y^{2}\right)+x^{2} y^{2} z-z^{4}$. It is easy to see that $S_{r}=S_{r}^{2} \cap\{f<0\}$ is homeomorphic to a union of two disjoint 2-discs, so that $H^{i}\left(S_{r}\right)=0$ for $i \geq 1$. As $\omega=z\left(x^{2}+y^{2}\right)$, then $\Omega$ is homeomorphic to $S^{1} \times(0,1)$, and so $H^{1}(\Omega) \neq 0$. Hence $T(f)$ is infinite.

Corollary 5.6. If $\Omega \neq \emptyset$ and the Euler-Poincaré characteristic $\chi(\Omega) \leq 0$, then $T(f)$ is infinite.

Remark 5.7. If $\omega$ is a quadratic form which may be reduced to the diagonal form $-x_{1}^{2}-\cdots-x_{i+1}^{2}+x_{i+2}^{2}+\cdots+x_{j}^{2}$, where $i \geq 1$, then the dimension of the stable manifold at the origin is at least two. Hence $T(f)$ is infinite.

Investigating the gradient flow in polar coordinates and applying arguments presented by Moussu in [18, p.449] the reader may also prove the next proposition. (As its proof would require to introduce other techniques, so we omit it here.)

Proposition 5.8. Suppose that there exists a non-degenerate critical point of $\omega \mid \Omega$ which is not a local minimum. Then $T(f)$ is infinite.

In particular, if there exists a non-degenerate local maximum of $\omega \mid \Omega$ then the interior of the stable set of the origin is non-empty.

Example 5.9. Let $f(x, y)=x^{3}+3 x y^{2}+x^{2} y^{2}$, so that $\omega=x^{3}+3 x y^{2}$. It is easy to see that $\omega \mid S^{1}$ has a non-degenerate local maximum at $(-1,0) \in \Omega$. Then the interior of the stable set of the origin is non-empty. In particular $T(f)$ is infinite.

The next theorem is the main result of this section.

Theorem 5.10. Suppose that $f: \mathbb{R}^{n}, 0 \rightarrow \mathbb{R}, 0$ is an analytic function having a critical point at the origin

If rank $H^{0}\left(S_{r}\right)<\operatorname{rank} H^{0}(\Omega)$, i.e. the number of connected components of $S_{r}$ is smaller than the number of connected components of $\Omega$, then the set of trajectories of the gradient flow $\dot{x}=\nabla f(x)$ converging to the origin is infinite.

Proof. Suppoose, contrary to our claim, that $T(f)$ if finite. Then $\Gamma(f)$ is finite, and for any $(\ell, a) \in \mathbb{Q}^{+} \times \mathbb{R}_{-} \backslash L^{\prime}(f)$ the set $\tilde{\Gamma}^{\ell, a}(f)$ is finite too. Hence $\operatorname{rank} \bar{H}^{0}\left(\tilde{\Gamma}^{\ell, a}(f)\right)$ equals the number of elements in $\tilde{\Gamma}^{\ell, a}(f)$.

By Lemma 3.4, there exist $0<r \ll-a \ll 1$ such that $\Omega$ and $S_{r}^{d, a}$ have the same homotopy type. By Corollary 5.2 , the group $H^{*}\left(S_{r}\right)$ is isomorphic 
to $\bar{H}^{*}(\Gamma(f))$. Hence $\operatorname{rank} H^{0}\left(S_{r}\right)=\operatorname{rank} \bar{H}^{0}(\Gamma(f))$ equals the number of elements in $\Gamma(f)$. Moreover, $\operatorname{rank} H^{0}(\Omega)=\operatorname{rank} H^{0}\left(S_{r}^{d, a}\right)=\operatorname{rank} \bar{H}^{0}\left(\tilde{\Gamma}^{d, a}(f)\right)$ equals the number of elements in $\tilde{\Gamma}^{d, a}(f)$.

As $\tilde{\Gamma}^{d, a}(f) \subset \Gamma(f)$, then $\operatorname{rank} H^{0}(\Omega) \leq \operatorname{rank} H^{0}\left(S_{r}\right)$, which contradicts the assumption.

Theorem 5.11. If $\chi\left(S_{r}\right)<\chi(\Omega)$ then $T(f)$ is infinite.

Proof. By Corollary 5.2 and Proposition 5.4, it is enough to consider the case where all cohomology groups $H^{i}\left(S_{r}\right), H^{i}(\Omega)$, where $i \geq 1$, are trivial.

If that is the case then $\operatorname{rank} H^{0}\left(S_{r}\right)=\chi\left(S_{r}\right)<\chi(\Omega)=\operatorname{rank} H^{0}(\Omega)$. By Theorem 5.10 , the set $T(f)$ is infinite.

Example 5.12. Let $f(x, y)=x^{3}-y^{2}$, so that $\omega=-y^{2}$. Then $\Omega=\{(x, y) \in$ $\left.S^{1} \mid-y^{2}<0\right\}=S^{1} \backslash\{( \pm 1,0)\}$. Obviously $\Omega$ has two connected components and $H^{i}(\Omega)=0$ for any $i \geq 1$. The function $\omega \mid \Omega$ has exactly two critical (minimum) points at $(0, \pm 1)$, so one cannot apply Proposition 5.8 .

As $S_{r}$ is homeomorphic to an interval, then by Theorem5.10 the set $T(f)$ is infinite.

Example 5.13. Let $f(x, y, z)=x y z-z^{4}$, so that $\omega=x y z$. It is easy to see that $\Omega$ is homeomorphic to a disjoint union of four discs, and $S_{r}$ is homeomorphic to a disjoint union of two discs. By Theorem 5.10 the set $T(f)$ is infinite.

Example 5.14. Let $f(x, y, z)=x y z+x^{4} y-2 y^{4} z+3 x z^{4}$, so that $f$ has an isolated critical point at the origin and $\omega=x y z$. Applying Andrzej Eȩcki computer program (see [15]) we have verified that the local topological degree of the mapping

$$
\mathbb{R}^{3}, 0 \ni(x, y, z) \mapsto-\nabla f(x, y, z) \in \mathbb{R}^{3}, 0
$$

equals zero. By [10], [11], the Euler-Poincaré characteristic $\chi\left(S_{r}^{2} \cap\{f \geq\right.$ $0\})=1-0=1$. By the Alexander duality theorem $\chi\left(S_{r}\right)=1$. By Theorem 5.11 the set $T(f)$ is infinite.

\section{Interior of the stable set.}

In this section we shall present simple topological conditions which imply that the interior of the stable set $S(f)$ has a non-empty interior 
The set $\Omega$ is semi-algebraic, hence $\operatorname{rank} H^{n-2}(\Omega)<\infty$. By Theorem 4.1 and Proposition 5.4, if $0<-a \ll 1$ then $\tilde{\Gamma}^{d, a}(f)$ is compact and $\operatorname{rank} \bar{H}^{n-2}\left(\tilde{\Gamma}^{d, a}(f)\right)<\infty$.

Remark 6.1. If $\omega$ is a quadratic form which can be reduced to the diagonal form $-x_{1}^{2}-\cdots-x_{i+1}^{2}+x_{i+2}^{2}+\cdots+x_{j}^{2}$, where $i \geq 1$, then

$$
\bar{H}^{*}\left(\tilde{\Gamma}^{d, a}(f)\right) \simeq H^{*}(\Omega) \simeq H^{*}\left(S^{i}\right) .
$$

In that case rank $\bar{H}^{n-2}\left(\tilde{\Gamma}^{d, a}(f)\right)=\operatorname{rank} H^{n-2}\left(S^{i}\right)>0$ if and only if $\omega$ can be reduced to the diagonal form $-x_{1}^{2}-\cdots-x_{n-1}^{2}$.

The next theorem is the main result of this section.

Theorem 6.2. Suppose that $f: \mathbb{R}^{n}, 0 \rightarrow \mathbb{R}, 0, n \geq 2$, is an analytic function defined in an open neighbourhood of the origin. Suppose that rank $H^{n-2}\left(S_{r}\right)<$ rank $H^{n-2}(\Omega)$. Then the stable set of the origin of the gradient flow $\dot{x}=$ $\nabla f(x)$ has a non-empty interior.

Proof. By [17, Lemma 5.10], if $0<-y \ll r \ll 1$ then the Milnor number $F_{r}(y)$ is homeomorphic to an $(n-1)$-dimensional submanifold of $S_{r}^{n-1}$.

As $\tilde{\Gamma}^{d, a}(f) \subset \Gamma(f)$ are compact subsets of $F_{r}(y)$ with $\operatorname{rank} \bar{H}^{n-2}(\Gamma(f))=$ $\operatorname{rank} H^{n-2}\left(S_{r}\right)<\operatorname{rank} H^{n-2}(\Omega)=\operatorname{rank} \bar{H}^{n-2}\left(\tilde{\Gamma}^{d, a}(f)\right)<\infty$, then by Corollary 2.2 the set $\Gamma(f)$ has a non-empty interior in $F_{r}(y)$.

Trajectories of the flow $\dot{x}=\nabla f(x)$ converging to the origin cut transversally $F_{r}(y)$ at point of $\Gamma(f)$. Hence the stable set of the origin has a nonempty interior.

Put $\Omega^{\prime}=S^{n-1} \cap\{\omega \geq 0\}=S^{n-1} \backslash \Omega$, and $S_{r}^{\prime}=S_{r}^{n-1} \cap\{f \geq 0\}=S_{r}^{n-1} \backslash S_{r}$, $0<r \ll 1$. Sets $\Omega^{\prime}, S_{r}^{\prime}$ are compact and semianalytic, hence they are triagulable. By the Alexander duality theorem, if $S_{r}^{\prime}$ and $\Omega^{\prime}$ are non-empty then $\operatorname{rank} H_{0}\left(S_{r}^{\prime}\right)=1+\operatorname{rank} H^{n-2}\left(S_{r}\right)$ and $\operatorname{rank} H_{0}\left(\Omega^{\prime}\right)=1+\operatorname{rank} H^{n-2}(\Omega)$.

Theorem 6.3. Suppose that the set $S_{r}^{\prime}$ has less connected components than $\Omega^{\prime}$. Then the stable set of the origin of the gradient flow $\dot{x}=\nabla f(x)$ has a non-empty interior.

Proof. The set $\Omega^{\prime}$ is obviously not empty. If $S_{r}^{\prime}=\emptyset$ then the origin is a strict local maximum, and then int $S(f) \neq \emptyset$.

Suppose that $S_{r}^{\prime} \neq \emptyset$. Sets $S_{r}^{\prime}, \Omega^{\prime}$ are compact, semianalytic. So they are triangulable, and the number of connected components of $S_{r}^{\prime}$ (resp. $\Omega^{\prime}$ ) equals the number of its path-components which is rank $H_{0}\left(S_{r}^{\prime}\right)\left(\operatorname{resp} . \operatorname{rank} H_{0}\left(\Omega^{\prime}\right)\right)$. 
By assumption, rank $H_{0}\left(S_{r}^{\prime}\right)<\operatorname{rank} H_{0}\left(\Omega^{\prime}\right)$ and then rank $H^{n-2}\left(S_{r}\right)<$ rank $H^{n-2}(\Omega)$. By Theorem 6.2, the stable set $S(f)$ has a non-empty interior.

Example 6.4. Let $f(x, y)=x^{3}-y^{2}$ be the same as in Example 5.12. Then $\Omega^{\prime}=\{(-1,0),(1,0)\}$. As $S_{r}^{\prime}$ is homeomorphic to a closed interval, then by Theorem 6.3 the interior of $S(f)$ is non-empty.

Example 6.5. Let $f(x, y, z)=-x^{2} y^{2}-z^{4}+x^{5}$. Then $\omega=-x^{2} y^{2}-z^{4}$ and $\Omega^{\prime}$ consists of four points. It is easy to see that $S_{r}^{\prime}$ is homeomorphic to a disjoint union of a closed disc and two points. By Theorem 6.3 the interior of $S(f)$ is non-empty.

\section{Right-equivalent functions}

Let $g: \mathbb{R}^{n}, 0 \rightarrow \mathbb{R}, 0$ be an analytic function which is right-equivalent to $f$, i.e. there exists a $C^{\infty}$-diffeomorphism $\phi: \mathbb{R}^{n}, 0 \rightarrow \mathbb{R}^{n}, 0$ defined in an open neighbourhood of the origin such that $g=f \circ \phi$. Then in particular the derivative $D \phi(0): \mathbb{R}^{n} \rightarrow \mathbb{R}^{n}$ is a linear isomorphism.

Let $\theta$ be the initial homogeneous form associated with $g$, let $\Theta=S^{n-1} \cap$ $\{\theta<0\}$, and let $\Theta^{\prime}=S^{n-1} \cap\{\theta \geq 0\}$. It is easy to see that $\theta=\omega \circ D \phi(0)$. Hence sets $\Omega$ and $\Theta$, as well as $\Omega^{\prime}$ and $\Theta^{\prime}$, are homeomorphic. Then $H^{0}(\Omega) \simeq$ $H^{0}(\Theta)$ and $H_{0}\left(\Omega^{\prime}\right) \simeq H_{0}\left(\Theta^{\prime}\right)$.

Both $f$ and $g$ are analytic, hence there exists small $r_{0}>0$ such that for each $0<r \leq r_{0}$ the number of connected components of $S_{r}^{\prime}$ equals the number of connected components of $\left(B_{r}^{n} \backslash\{0\}\right) \cap\{f \geq 0\}$, and the the number of connected components of $S_{r}^{n-1} \cap\{g \geq 0\}$ equals the number of connected components of $\left(B_{r}^{n} \backslash\{0\}\right) \cap\{g \geq 0\}$. As $g=f \circ \phi$ then $\left(B_{r}^{n} \backslash\{0\}\right) \cap\{g \geq 0\}$ is homeomorphic to $\left(\phi\left(B_{r}^{n}\right) \backslash\{0\}\right) \cap\{f \geq 0\}$.

There exist $0<r_{3}<r_{2}<r_{1}<r_{0}$ such that $\phi\left(B_{r_{3}}^{n}\right) \subset B_{r_{2}}^{n} \subset \phi\left(B_{r_{1}}^{n}\right) \subset$ $B_{r_{0}}^{n}$.

The inclusion $\left(B_{r_{3}}^{n} \backslash\{0\}\right) \cap\{g \geq 0\} \subset\left(B_{r_{1}}^{n} \backslash\{0\}\right) \cap\{g \geq 0\}$ is a homotopy equivalence. Hence inclusions

$$
\begin{gathered}
\left(\phi\left(B_{r_{3}}^{n}\right) \backslash\{0\}\right) \cap\{f \geq 0\} \subset\left(\phi\left(B_{r_{1}}^{n}\right) \backslash\{0\}\right) \cap\{f \geq 0\} \\
\left(B_{r_{2}}^{n} \backslash\{0\}\right) \cap\{f \geq 0\} \subset\left(B_{r_{0}}^{n} \backslash\{0\}\right) \cap\{f \geq 0\}
\end{gathered}
$$


are homotopy equivalencies, and then in particular sets $\left(B_{r_{1}}^{n} \backslash\{0\}\right) \cap\{g \geq 0\}$, $\left(\phi\left(B_{r_{1}}^{n}\right) \backslash\{0\}\right) \cap\{f \geq 0\}$ and $\left(B_{r_{0}}^{n} \backslash\{0\}\right) \cap\{f \geq 0\}$ have the same number of connected components.

Hence sets $S_{r}^{\prime}$ and $S_{r}^{n-1} \cap\{g \geq 0\}$ have the same number of connected components too. By similar arguments, the sets $S_{r}$ and $S_{r}^{n-1} \cap\{g<0\}$ have the same number of connected components too. By Theorems [5.10, 6.3 we get

Theorem 7.1. Suppose that an analytic function $g: \mathbb{R}^{n}, 0 \rightarrow \mathbb{R}, 0$ is rightequivalent to $f$. If $S_{r}$ has less connected components than $\Omega$ then $T(g)$ is infinite. If $S_{r}^{\prime}$ has less connected components than $\Omega^{\prime}$ then $S(g)$ has a nonempty interior.

The next example demonstrates that the assumptions of Theorem 7.1 are significant.

Example 7.2. Let $f(x, y)=x^{3}+3 x y^{2}$, so that $S_{r}^{\prime}$ and $\Omega^{\prime}$ are homeomorphic. The same way as in Example 5.9 one can show that the interior of $S(f)$ is non-empty. The function $g(x, y)=f(\sqrt{3} x, y)=3 \sqrt{3}\left(x^{3}+x y^{2}\right)$ is right-equivalent to $f$. Applying the polar coordinates one can show that $S(g)$ consists of a single trajectory, so that its interior is empty.

In the case where $g$ has an algebraically isolated critical point at the origin, one can compute its Milnor number $\mu(g)=\operatorname{dim}_{\mathbb{R}} \mathbb{R}\left[\left[x_{1}, \ldots, x_{n}\right]\right] /\langle\partial g\rangle$, where $\langle\partial g\rangle$ is the ideal in $\mathbb{R}\left[\left[x_{1}, \ldots, x_{n}\right]\right]$ generated by $\partial g / \partial x_{1}, \ldots, \partial g / \partial x_{n}$ (see [17]).

Theorem 7.3. Let $g: \mathbb{R}^{n}, 0 \rightarrow \mathbb{R}, 0$ be an analytic function having an algebraically isolated critical point at the origin. Suppose that $\mu(g)$ is even, and $\theta$ is a quadratic form which can be reduced to the diagonal form $-x_{2}^{2}-\cdots-x_{n}^{2}$. Then the interior of $S(g)$ is non-empty.

Proof. Applying standard methods of the singularities theory (see [3]) one can show that $g$ is right-equivalent to $f=x_{1}^{k}-x_{2}^{2}-\ldots-x_{n}^{2}$, where $k=\mu(g)+1$. Then $S_{r}^{\prime}$ is homeomorphic to a closed $(n-2)$-dimensional closed ball and $\Omega^{\prime}$ consists of two points. By Theorem 7.1 , the set $S(g)$ has a non-empty interior.

Example 7.4. Let $g(x, y, z, w)=x^{5}+z^{5}+2 z w-x^{2}-y^{2}-z^{2}-w^{2}-2 x y z-y^{2} z^{2}$. In this case $\mu(g)=4$, and $\theta$ can be reduced to the diagonal form $-y^{2}-z^{2}-w^{2}$. By Theorem 7.3, the set $S(g)$ has a non-empty interior. 


\section{References}

[1] P.-A. Absil, K. Kurdyka, On the stable equilibrium points of gradient systems, Systems \& Control Letters 55 (2006) 573-577.

[2] C. Böhm, R. Lafuente, M. Simon, Optimal curvature estimates for homogeneous Ricci flows, Int. Math. Res. Not. IMRN 14 (2019) 4431-4468.

[3] Th. Bröcker, L. Lander, Differentiable germs and catastrophes, Cambridge University Press, 1975.

[4] A. Dzedzej, Z. Szafraniec, On families of trajectories of an analytic gradient vector field, Ann. Polon. Math. 87 (2005) 99-109.

[5] R. Engelking, Outline of general topology. Translated from the Polish by K. Sieklucki North-Holland Publishing Co., Amsterdam; PWN-Polish Scientific Publishers, Warsaw; Interscience

[6] P. Goldstein, Gradient flow of a harmonic function in $\mathbb{R}^{3}$, J. Differential Equations 247 (9) (2009) 2517-2557.

[7] M. Goresky, R. MacPherson, Stratified Morse theory, Springer-Verlag Berlin Heidelberg, 1988.

[8] R. Hardt, Stratification of real analytic mappings and images, Invent. Math. 28 (1975) 193-208.

[9] H. Hironaka, Stratification and flatness. Real and Complex Singularities, Proc. Oslo 1976, Sijthoffand Noordhoff (1977), pp. 199-265.

[10] G. M. Khimshiashvili, On the local degree of a smooth mapping, Comm. Acad. Sci. Georgian SSR. 85 (1977) 309-311 (in Russian).

[11] G. M. Khimshiashvili, On the local degree of a smooth mapping. Trudy Tbilisi Math. Inst. 64 (1980) 105-124.

[12] K. Kurdyka, T. Mostowski, A. Parusiński, Proof of the gradient conjecture of R. Thom, Ann. of Math. 152 (2000) 763-792.

[13] K. Kurdyka, A. Parusiński, Quasi-convex decomposition in o-minimal structures. Application to the gradient conjecture, Advanced Studies in Pure Mathematics 43 (2006), Singularity Theory and Its Applications, pp. 137-177. 
[14] C. Lageman, Convergence of gradient-like dynamical systems and optimization algorithms, Ph.D. Thesis, University of Würzburg, 2007.

[15] A. Eȩcki, Z. Szafraniec, Applications of the Eisenbud \& Levine's theorem to real algebraic geometry, Computational Algebraic Geometry, Progr. Math. 109, Birkhäuser 1993, 177-184.

[16] S. Łojasiewicz, Sur les trajectoires du gradient d'une fonction analytique, Seminari di Geometria 1982-1983, Università di Bologna, Istituto di Geometria, Dipartamento di Matematica (1984) 115-117.

[17] J. Milnor, Singular points of complex hypersurfaces, Annals of Mathematics Studies 61, Princeton Univ. Press, Princeton, NJ, 1968.

[18] R. Moussu, Sur la dynamique des gradients. Existence de varietes invariantes, Math. Ann. 307 (1997) 445-460.

[19] A. Nowel, Z. Szafraniec, On trajectories of analytic gradient vector fields, J. Differential Equations 184 (2002) 215-223.

[20] A. Nowel, Z. Szafraniec, On trajectories of analytic gradient vector fields on analytic manifolds, Topol. Methods Nonlinear Anal. 25 (2005) 167182.

[21] E. H. Spanier, Algebraic topology, McGraw-Hill, New York, 1966.

[22] Z. Szafraniec, Topological invariants of weighted homogeneous polynomials, Glasgow Math. J. 33 (1991) 241-245.

[23] Z. Szafraniec, On the family of trajectories of an analytic gradient flow converging to a critical point, http://arxiv.org/abs/1912.09149.

\section{Zbigniew SZAFRANIEC}

Institute of Mathematics, University of Gdańsk

80-952 Gdańsk, Wita Stwosza 57, Poland

Zbigniew.Szafraniec@mat.ug.edu.pl 